\title{
Can Number of Sites of Obstruction Be Predicted on a Level 3 Home Sleep Study?
}

\author{
Dasari Samuel Deenadayal*, Dumpala Vidyasagar, Vyshanavi Bommakanti \\ Department of ENT and HNS, Deenadayal ENT Care Centre, Secunderabad, India \\ Email: *vyshnavi.jajee@gmail.com
}

How to cite this paper: Deenadayal, D.S. Vidyasagar, D. and Bommakanti, V. (2018) Can Number of Sites of Obstruction Be Predicted on a Level 3 Home Sleep Study? International Journal of Otolaryngology and Head \& Neck Surgery, 7, 80-87. https://doi.org/10.4236/ijohns.2018.73011

Received: March 9, 2018

Accepted: May 7, 2018

Published: May 10, 2018

Copyright $\odot 2018$ by authors and Scientific Research Publishing Inc. This work is licensed under the Creative Commons Attribution International License (CC BY 4.0).

http://creativecommons.org/licenses/by/4.0/

\begin{abstract}
Objective: To assess the number of levels of obstruction on a level 3 home sleep study in patients with obstructive sleep apnea. Study Design: This is a prospective study. Setting: Tertiary referral centre. Subjects and Methods: All the patients with the complaints of snoring, excessive daytime sleepiness, were evaluated for OSA. Complete history was taken and a thorough clinical examination was done followed by fibreoptic nasopharyngoscopy to determine the sites of obstructions. Patients were categorised into group A and group B based on the number of levels of obstruction. A level 3 home sleep study also was done for these patients. We tried to correlate the polysomnographic variables between the two groups. Results: There was a significant difference between the two groups in a number of PSG parameters. Patients in A group had lower number of apnoeas, obstructive apnoeas, hypopneas, AHI index, flow limitation and BMI as compared to patients in group B and the difference was statistically significant. We have devised a score using 3 parameters AHI, Flow limitation with snoring and BMI, the Deenadayal's (DDS) scoring system. The minimum scoring would be 3 and maximum score would be 6 . Based on scoring the probable number of obstructions can be identified. Conclusion: Sleep study report can yield a lot of information regarding the number of levels of obstruction. A critical analysis is required while doing the same. Till date there have been no reports in literature identifying number of levels of obstruction on PSG.
\end{abstract}

\section{Keywords}

Home Sleep Study, Levels of Obstruction

\section{Introduction}

Obstructive sleep apnea (OSA) is a sleep-related breathing disorder that involves 
a decrease or complete halt in airflow despite an ongoing effort to breathe. It occurs as a result of soft tissue collapse at the nasopharynx or the oropharynx causing airway obstruction resulting in hypopnea and apnea. It has been proposed that the pathophysiological pathways linking these risk factors for OSA can be explained by anatomical abnormalities, increased pharyngeal dilator muscle dysfunction, lowered arousal threshold, increased ventilatory control instability, and/or reduced lung volume [1].

Patients with obstructive sleep apnea demonstrate oropharyngeal and hypopharyngeal wall collapse during inspiration. Patients with obstructive sleep apnea who do not have isolated obstructive lesions in the upper airway must have some dynamic alteration that allows the relatively hypotonic pharyngeal walls to collapse into the inspiratory airstream. This disproportionate anatomy, consisting of any combination of large tongue base, long soft palate, shallow palatal arch narrow mandibular arch or retrognathic mandible, renders the patient more vulnerable to the development of relative upper airway obstruction. This alteration causes intraluminal pressure to become excessively negative and allows the underlying hypotonic pharyngeal walls to collapse [2].

The symptoms of OSA include snoring unrefreshed sleep, excessive daytime sleepyness, and reduced concentration. OSA is a risk factor for hypertension, diabetes mellitus and it can have long term consequences like stroke and myocardial infarction.

The gold standard test for the diagnosis of obstructive sleep apnea is polysomnography. In addition fibreoptic nasopharyngoscopy, sleep endoscopy and dynamic MRI help in identifying the level of obstruction.

In our study we conducted polysomnography on all the candidates who were suspected as OSA based on symptoms and clinical examination. The findings observed in the sleep study were correlated with the levels of the obstruction.

\section{Materials and Methods}

The aim of the study is to correlate polysomnography variables with number of levels of obstruction identified on clinical examination and fibreoptic nasopharyngoscopy.

This study was conducted in the department of Yashoda Hospital Secunderabad from March 2014 to March 2015. Institute's Ethical committee clearance has been taken to conduct this study.

All the patients with the complaints of snoring, excessive daytime sleepiness, unintentional sleeping episodes during wakefulness, reduced concentration, unrefreshing sleep, fatigue, insomnia were evaluated for Ostructive sleep apnea. Complete history was taken from the patient as well as patients' bed partner. Any witnessed Apnea observed by the bed partner was asked for specifically. The anthropometric data of patients i.e. height, weight, body mass index was documented. All the patients were asked to grade themselves on the Epworth sleepyness Scale. All the patients underwent clinical examination and fibreoptic laryngopharyngoscopy with muller manoeuvre to identify the site of obstruction. The 
fibreoptic evaluation was performed by our team of qualified otorhinolaryngologists, always under supervision of the senior author. These patients also underwent sleep study.

These patients were classified into various groups depending on the number of levels of obstruction.

\begin{tabular}{cc}
\hline Group & Obstruction \\
\hline Group 1-Single level & Nose/Nasopharynx or \\
& Palate or \\
& Tongue base \\
Group 2-Two levels & Nose + palate or \\
& Nose + tongue base or \\
Group 3-Three levels & Palate + tongue base \\
\hline
\end{tabular}

Patients of Group 1 and 2 were labelled as A and Group 3 labelled as B. A comparative analysis was done using SPSS software between A and B using one way ANOVA test.

\subsection{Inclusion Criteria}

The diagnosis of OSA was made according to the criteria developed by American academy of sleep medicine. At least 1 of the following criteria must apply for OSA to be diagnosed:

1) The patient reports daytime sleepiness, unrefreshing sleep, fatigue, insomnia, and/or unintentional sleep episodes during wakefulness. The patient awakens with breath holding, gasping, or choking. The patient's bed partner reports loud snoring, breathing interruptions, or both during the patient's sleep.

2) Polysomnography (PSG) shows more than 5 scoreable respiratory events per hour of sleep and/or evidence of respiratory effort during all or a portion of each respiratory event.

3) PSG shows more than 15 scorable respiratory events per hour of sleep and/or evidence of respiratory effort during all or a portion of each respiratory event.

4) Another current sleep disorder, medical or neurologic disorder, medication use, or substance use does not better account for the patient's condition.

All the patients meeting at least one of the above criteria were diagnosed to be suffering from OSA and were included from the study and all the other patients were excluded.

\subsection{Exclusion Criteria}

- Paediatric patients

- Patients with insomnia

\section{Results}

A total of 196 polysomnoraphic reports were evaluated. In 10 patients complete 
data was not available and were excluded, hence the sample size was 186.

Males in our study were 143 while females were 43 hence the sex ratio was....

The mean neck circumference was 15.5 inches.

The observations are as shown in Table 1.

As seen in the table above, there was a significant difference between the two groups in a number of PSG parameters. Patients in A group had lower number of apnoeas, obstructive apnoeas, hypopneas, AHI index, flow limitation with snoring scores, total snoring events, lowest oxygen saturation and BMI compared to patients in group B and the difference was statistically significant. We have devised score was devised using 3 parameters AHI, Flow limitation with snoring and BMI. The Deenadayal's (DDS) scoring system is shown in Table 2.

The minimum score would be 3 and maximum score was 6 . We have analysed the difference in DDS score between A and B groups using Chi-Square test (Table 3 ). The difference was significant with a p-value of 0.006 , which is

Table 1. A vs B (ANOVA).

\begin{tabular}{|c|c|c|c|c|c|c|c|}
\hline & \multicolumn{2}{|c|}{1 or 2 level obstruction. $(n=80)$} & \multicolumn{2}{|c|}{3 level obstruction. $(n=106)$} & \multicolumn{2}{|c|}{ Total } & \multirow{2}{*}{$\begin{array}{c}\text { ANOVA } \\
\text { Sig. }\end{array}$} \\
\hline & Mean & Std. Deviation & Mean & Std. Deviation & Mean & Std. Deviation & \\
\hline Age & 45.73 & 12.460 & 46.23 & 9.736 & 46.02 & 10.967 & 0.75 \\
\hline Apnea & 54.96 & 92.795 & 186.60 & 195.080 & 129.79 & 171.782 & $<0.02$ \\
\hline Obstructive & 51.56 & 86.991 & 175.38 & 184.683 & 121.94 & 162.277 & $<0.02$ \\
\hline Hypopnea & 62.22 & 51.005 & 86.91 & 57.147 & 76.25 & 55.805 & $<0.02$ \\
\hline AHI & 19.5305 & 19.01599 & 45.5849 & 34.19689 & 34.3404 & 31.37583 & $<0.02$ \\
\hline Flow Limitation with Snoring & 1199.96 & 908.270 & 1520.66 & 1045.878 & 1338.37 & 980.407 & 0.02 \\
\hline Flow Limitation without Snoring & 563.45 & 683.548 & 691.09 & 523.203 & 636.01 & 599.365 & 0.14 \\
\hline Average Saturation & 94.293 & 3.5154 & 93.287 & 3.4181 & 93.721 & 3.4871 & 0.14 \\
\hline Lowest Saturation & 81.27 & 9.177 & 75.29 & 10.568 & 77.87 & 10.399 & $<0.02$ \\
\hline Baseline Saturation & 96.04 & 2.631 & 96.65 & 1.585 & 96.39 & 2.120 & 0.14 \\
\hline Body Mass Index & 25.2446 & 4.89654 & 33.8202 & 6.13789 & 29.5726 & 5.63049 & $<0.02$ \\
\hline
\end{tabular}

Table 2. Deenadayal's scoring system.

\begin{tabular}{ccc} 
& Parameter & Score \\
\hline AHI & $\leq 48.5$ & 1 \\
& $>48.5$ & 2 \\
BMI & $\leq 29$ & 1 \\
Flow limitation with snoring & $>29$ & 2 \\
& $\leq 1300$ & 1 \\
\hline
\end{tabular}

Apnea Hypopnea Index (AHI): The AHI is the number of apneas or hypopneas recorded during the study per hour of sleep. Body Mass Index (BMI) is a person's weight in kilograms (kg) divided by his or her height in meters squared. 
Table 3. A vs B * DDS score Chisquare test.

\begin{tabular}{|c|c|c|c|c|c|c|c|c|}
\hline & & & \multicolumn{4}{|c|}{ dds3 } & \multirow{2}{*}{ Total } & \multirow{2}{*}{$\begin{array}{l}\text { Asymp. Sig } \\
\text { (2-sided) }\end{array}$} \\
\hline & & & 3.00 & 4.00 & 5.00 & 6.00 & & \\
\hline \multirow{5}{*}{ A vs $B$} & 1 or 2 level & Count & 25 & 34 & 21 & 0 & 80 & \multirow{5}{*}{0.006} \\
\hline & obstruction & $(\%)$ & $67.6 \%$ & $35.1 \%$ & $41.2 \%$ & $0.0 \%$ & $43.0 \%$ & \\
\hline & 3 or more than & Count & 12 & 63 & 30 & 1 & 106 & \\
\hline & 3 level obstruction & $(\%)$ & $32.4 \%$ & $64.9 \%$ & $58.8 \%$ & $100.0 \%$ & $57.0 \%$ & \\
\hline & Total & Count & 37 & 97 & 51 & 1 & 186 & \\
\hline
\end{tabular}

statistically significant. Thus, lower the score the probability of single site or two sites obstruction is higher while higher the score the chances of obstruction at three sites is high.

This scoring can be used to score all polysomgraphic reports and add to the information regarding the number of level of obstruction.

\section{Discussion}

Obstructive sleep apnea is a syndrome of upper airway collapse with an increased effort to breathe. There is an increase in the prevalence of OSA in the last few decades. Obstructive sleep apnea (OSA) affects at least $2 \%$ to $4 \%$ of the adult population and is increasingly being recognized by the public [3].

Obstructive sleep apnoea disturbs the quality of sleep and has number of immediate and longterm complications. It disturbs sleep and causes excessive day time sleepiness. This seriously impairs ones quality of life and working ability. Neglected OSA leads to resistant hypertension, diabetes and serious cardiopulmonary complications like arrhtymias, pulmonary hypertension, cor-pulmonale and death [4]. The incidence of OSA with stroke is reported to be between $50 \%$ to 70\% [5]. Various studies have showed occurrence of OSA and Pulmonary fibrosis to be $88 \%$ [6] [7] [8]. OSA is a chronic condition hence patient education is extremely important in these patients

OSA is diagnosed clinically and is aided by a number of investigations. A number of surgies are being done with good clinical results. Identifying the level of obstruction is a key factor in choosing the surgical approach. A number of investigations like polysomnography, Drug induced sleep endoscopy, Sleep MRI and various other investigations are helpful in indentifying the level of obstruction. In a developing country like India where the patient can afford to have only a few tests, getting most of the information from one test is extremely useful. Polysomnogram is the basic investigation for diagnosis and management of OSA. It is considered as a "gold-standard" [3]. As it is most commonly done investigation, we have evaluated its role in identifying the number of levels of obstruction in our study.

Polysomnography studies are an essential tool for the sleep physician and aid in the diagnosis and treatment of sleep disorders. The term polysomnography 
refers to the recording, analysis, and interpretation of multiple physiologic signals collected simultaneously. Typical physiologic signals that are collected as part of the polysomnography study include, but are not limited to, electroencephalogram (EEG), electromyogram (EMG), electro-oculogram (EOG), electrocardiogram (ECG), and respiratory signals.

A total of 186 patients were included in our study. Out of 186 patients, 157 (84.4\%) were male patients and 29 (15.6) were female patients. This male preponderance was also found in a Singapore review of PSG. There were 449 male patients $(76.9 \%)$ and 135 female patients $(23.1 \%)$, with a mean age of 47.5 years (SD 12.7) [9]. The Wisconsin Sleep Cohort Study, the largest reported prevalence study with PSG among middle-aged adults, has reported an estimated prevalence of OSAS (AHI $\geq 5$ and excessive daytime sleepiness) of $2 \%$ in women, and $4 \%$ in men. In this study, male gender and obesity were strongly associated with the presence of sleep-disordered breathing [10].

The mean BMI in our study was 25.24 for patients with one or two levels of obstruction and 33.82 for patients with greater than 2 levels of obstruction. The difference was statistically significant. Thus, we have found a significant correlation between BMI and the number of levels of obstruction in our study. A number of studies have found a strong association between OSA and BMI [10]. A few authors have correlated between the PSG parameters and BMI. Lim et al, Dixon et al. and Rauf et al. have found a fair degree of correlation between AHI and BMI, with more severe OSA patients tending to have a higher BMI [9] [11] [12].

In a smaller patient cohort consisting of 87 patients, Ozdas et al. found a significant correlation between BMI and AHI, and similarly suggested that increased BMI with obesity caused increased fat deposition in the lateral pharyngeal regions causing collapse of lateral pharyngeal walls both in oropharyx and hypopharynx on muellers manoeuvre [13]. In OSAS patients, increased BMI and $\mathrm{NC}$ are correlated with lateral pharyngeal wall collapse in retropalatal and retrolingual levels [11].

Inspiratory flow limitation (IFL) can be observed as flattening of the flow tracing on PSG. Physiologically, IFL indicates absence of an increase in flow despite an elevation in negative intrathoracic pressure indicating increasing effort [14]. Basically the importance of flow limitation lies in identifying other forms of sleep disordered breathing like upper airway resistance syndrome. Flow limitation without snoring is an indicator of probable nasal obstruction and flow limitation with snoring is an indicator of palatal and tongue base obstruction [14]. But in our study people with one or two level obstruction had lower flow limitation with snoring scores compared to patients with three levels of obstruction and the difference was statistically significant. The difference in flow limitation without snoring scores of the two groups was not statistically significant.

There are array of tests available for diagnosing and identifying the level of obstruction in these patients. We couldn't find literature addressing the role of PSG in identifying the number of level of obstruction. In this study we have tried 
to score the polysomnograph with 3 variables: AHI, Flow limitations with snoring and the body mass index. This score helps to predict number of level of obstruction. Thus, lower the score the probability of single site or two sites obstruction is higher while higher the score the chances of obstruction at three sites is high.

Since polysomnography remains the gold standard and the first line of investigation in each patient of obstructive sleep apnea an additional information regarding the number of sites of obstruction can be very helpful even before further testing.

This scoring system will be further utilized in the next prospective study of our institute to see how it actually works.

The scoring system can also help postoperatively in comparing the score preoperatively with that of post-operative score.

\section{Conclusion}

Sleep study report can yield a lot of information regarding the number of levels of obstruction. A critical analysis is required while doing the same.

\section{References}

[1] Eckert, D.J. and Malhotra, A. (2008) Pathophysiology of Adult Obstructive Sleep Apnea. Proceedings of the American Thoracic Society, 5, 144-153. https://doi.org/10.1513/pats.200707-114MG

[2] Rojewski, T.E., Schuller, D.E., Clark, R.W., Schmidt, H.S. and Potts, R.E. (1984) Videoendoscopic Determination of the Mechanism of Obstruction in Obstructive Sleep Apnea. Otolaryngology-Head and Neck Surgery, 92, 127-131. https://doi.org/10.1177/019459988409200201

[3] Epstein, L.J., Kristo, D., Strollo, P.J., et al. (2009) Clinical Guideline for the Evaluation, Management and Long-Term Care of Obstructive Sleep Apnea in Adults. Journal of Clinical Sleep Medicine, 5, 263-276.

[4] Davis, A.P., Billings, M.E., Longstreth, W.T. and Khot, S.P. (2013) Early Diagnosis and Treatment of Obstructive Sleep Apnea after Stroke: Are We Neglecting a Modifiable Stroke Risk Factor? Neurology Clinical Practice, 3, 192-201. https://doi.org/10.1212/CPJ.0b013e318296f274

[5] Hermann, D.M. and Bassetti, C.L. (2009) Sleep-Related Breathing and Sleep-Wake Disturbances in Ischemic Stroke. Neurology, 73, 1313-1322. https://doi.org/10.1212/WNL.0b013e3181bd137c

[6] Pihtili, A., Bingol, Z., Kiyan, E., Cuhadaroglu, C., Issever, H. and Gulbaran, Z. (2013) Obstructive Sleep Apnea Is Common in Patients with Interstitial Lung Disease. Sleep Breath, 17, 1281-1288. https://doi.org/10.1007/s11325-013-0834-3

[7] Mermigkis, C., Stagaki, E., Tryfon, S., et al. (2010) How Common Is Sleep-Disordered Breathing in Patients with Idiopathic Pulmonary Fibrosis? Sleep Breath, 14, 387-390. https://doi.org/10.1007/s11325-010-0336-5

[8] Lancaster, L.H., Mason, W.R., Parnell, J.A., Rice, T.W., Loyd, J.E., Milstone, A.P. and Collard, H.R. MB. (2009) Obstructive Sleep Apnea Is Common in Idiopathic Pulmonary Fibrosis. Chest, 136, 772-778. https://doi.org/10.1378/chest.08-2776

[9] Lim, L.L., Tham, K.W. and Fook-Chong, S.M.C. (2008) Obstructive Sleep Apnoea 
in Singapore: Polysomnography Data from a Tertiary Sleep Disorders Unit. Annals, Academy of Medicine, Singapore, 37, 629-636.

http://www.ncbi.nlm.nih.gov/pubmed/18797554

[10] Young, T., Palta, M., Dempsey, J., Skatrud, J., Weber, S. and Badr, S. (1993) The Occurrence of Sleep-Disordered Breathing among Middle-Aged Adults. The New England Journal of Medicine, 328, 1230-1235. https://doi.org/10.1056/NEJM199304293281704

[11] Kum, R.O., Ozcan, M., Yllmaz, Y.F., Gungor, V., Yurtsever Kum, N. and Unal, A. (2014) The Relation of the Obstruction Site on Muller's Maneuver with BMI, Neck Circumference and PSG Findings in OSAS. Indian Journal of Otolaryngology and Head \& Neck Surgery, 66, 167-172. https://doi.org/10.1007/s12070-014-0699-1

[12] Dixon, J.B., Schacter, L.M. and O’Brien, P.E. (2003) Predicting Sleep Apnea and Excessive Day Sleepiness in the Severely Obese: Indicators for Polysomnography. Chest, 123, 1134-1141. https://doi.org/10.1378/chest.123.4.1134

[13] Ozdas, T., Ozcan, K.M., Ozdogan, F., et al. (2013) Investigation of Lateral Pharyngeal Walls in OSAS. European Archives of Oto-Rhino-Laryngology, 270, 767-771. https://doi.org/10.1007/s00405-012-2212-3

[14] Sabil, A., Eberhard, A., Baconnier, P. and Benchetrit, G. (2004) A Physical Model of Inspiratory Flow Limitation in Awake Healthy Subjects. Advances in Experimental Medicine and Biology, 551, 211-216. https://doi.org/10.1007/0-387-27023-X_32 